Z Gerontol Geriat 2010 • 43:145-146

DOI 10.1007/s00391-010-0115-8

Online publiziert: 12. Mai 2010

(c) Springer-Verlag 2010

\author{
Th. Nikolaus \\ Geriatrische Klinik gGmbH, Universtität Ulm
}

\title{
Einheit in der Vielfalt
}

Im vorliegenden Heft wird ein großes Spektrum geriatrischer Fragestellungen präsentiert. Die untersuchten Problemstellungen sind häufig in unterschiedlicher Kombination bei einem geriatrischen Patienten anzutreffen. Die Multimorbidität erfordert eine ganzheitliche Betrachtungsweise des Menschen und macht gerade die Stärke der Altersmedizin aus: die Berücksichtigung verschiedener Erkrankungen mit ihrer komplexen Interaktion.

Gnass et al. führten eine systematische Literaturanalyse zum Thema „Erworbene Kontrakturen der Gelenke im höheren Lebensalter" durch. Insgesamt fanden die Wissenschaftler 37 kontrollierte Studien, die ihren Einschlusskriterien entsprachen. In nur 5 Publikationen wurde die Kontraktur präzise definiert. Die Autoren kommen in ihrer Bewertung zu dem Fazit, dass aufgrund der großen methodologischen Unterschiede, Diversität von Definitionen und Erhebungsmerkmalen die bisher durchgeführten Studien zu dieser Thematik miteinander nicht vergleichbar sind und keine allgemeingültigen Schlüsse gezogen werden können. Die Ergebnisse, die die Autoren gefunden haben, zeigen ein generelles Problem auf: die nicht einheitliche Definition medizinischer Fachtermini, die einen Vergleich verschiedener Studien so schwierig machen. Ein klassisches, häufig zitiertes Beispiel in der Altersmedizin ist hierfür die Definition des Sturzes.

Schwesig et al. untersuchten posturale Defizite und das Sturzrisiko bei älteren Personen mit Verdacht auf Osteoporose. Ziel der Untersuchung war es, die Prüfung eines neuen posturographischen Messsystems hinsichtlich Sturzprädiktion bei Osteoporosepatienten durchzuführen. Eingeschlossen in die Untersuchung wurden 228 Personen mit Osteoporoseverdacht, von denen 139 tatsächlich eine Osteoporose aufwiesen. Das neue posturographische Messsystem (Interaktives Balancesystem, IBS) erwies sich als leistungsfähiges und praktikables Assessment zur Prädiktion von Stürzen bei Osteoporosepatienten mit hohem Sturzrisiko. Das System lieferte darüber hinaus noch wichtige Informationen bezüglich defizitärer posturaler Subsysteme und kann daher auch zur Therapieplanung angewandt werden.

Ploenes et al. haben sich die Mühe gemacht, evidenzbasierte Empfehlungen zur Basisdiagnostik der peripheren arteriellen Verschlusskrankheit bei geriatrischen Patienten zu erarbeiten. Bei den Autoren handelt es sich um Mitglieder der Arbeitsgruppe Gefäßerkrankungen der Deutschen Gesellschaft für Geriatrie. Die Empfehlungen wurden in Delphi-Konferenzen erarbeitet, die Recherche der Primärliteratur erfolgte in den üblichen Datenbanken. Die Originalarbeiten wurden nach den Evidenzkriterien des Oxford Centre for Evidence-based Medicine eingestuft. Die Empfehlungen sind sehr praxisorientiert und geben dem geriatrisch tätigen Kollegen eine Empfehlung zur Basisdiagnostik der peripheren arteriellen Verschlusskrankheit.

Heidenblut $u$. Zank haben ein neues Depressionsscreening für den Einsatz in der Geriatrie entwickelt. Ausgangspunkt für die Entwicklung des neuen Messinstrumentes war die Ansicht der Autoren, dass die international sehr weit verbreitete „Geriatric Depression Scale“ (GDS) zwar sehr praktikabel, jedoch inhaltlich nur mit Einschränkungen auf die Zielgruppe einer geriatrischen Population zugeschnitten ist. In ihrer Untersuchung wurde das neue Instrument „Depression-im-Alter-Skala“ (DIA-S) an einer Stichprobe von 331 geriatrischen Patienten untersucht, von denen
151 depressive Symptome aufwiesen. Das neue Instrument wurde mit der GDS verglichen, wobei die Montgomery-AspergDepressionsskala (MADRS) als Goldstandardkriterium diente. Es zeigte sich eine hohe Praktikabilität des neuen Testinstrumentes sowie eine höhere interne Konsistenz und bessere Spezifität als die GDS15. Zur endgültigen Empfehlung sind jedoch weitere Validierungsstudien auch an ambulanten und teilstationären Patienten notwendig.

Honneth et al. schildern anhand eines Fallbeispiels den Stellenwert der molekularen Diagnostik bei Alzheimer-Demenz (AD). Schätzungsweise weniger als $5 \%$ aller Alzheimer-Erkrankungen manifestieren sich vor dem 66. Lebensjahr und werden als „early-onset Alzheimer disease“ (EO-AD) definiert. Bei den EO-AD kann es sich um genetisch vererbte Krankheitsbilder handeln. Die Autoren schildern in ihrer Kasuistik die Bedeutung der Familienanamnese bei präseniler Demenz (EO$\mathrm{AD})$ und des Einsatzes der molekulargenetischen Diagnostik in einer Demenzambulanz sowie die Konsequenzen des Befundes für die Angehörigenberatung.

Hewer $u$. Stark untersuchten retrospektiv bei 50 konsekutiv stationär behandelten gerontopsychiatrischen Patienten somatische Therapiemaßnahmen. Insgesamt wurden somatische Begleiterkrankungen bei $88 \%$ der Patienten diagnostiziert, bei drei Viertel der Patienten war eine Intervention auf somatischem Gebiet erforderlich. Dabei handelte es sich insbesondere um respiratorische und Harnwegsinfektionen sowie Herz-Kreislauf-Erkrankungen, gefolgt von metabolischen Störungen. Dies unterstreicht erneut, wie wichtig gerade im höheren Lebensalter eine enge Verzahnung gerontopsychiatrischer und geriatrischer Be- 
handlung ist, da auch im umgekehrten Falle viele primär geriatrisch zu behandelnde Patienten gerontopsychiatrische Symptome aufweisen.

Zum Thema „Pflegerische Betreuungskonzepte bei Patienten mit Demenz" untersuchten Nocon et al. in einem systematischen Review, welche pflegerischen Konzepte, wie die multisensorische Stimulation, die Validation, die Realitätsorientierung und Reminiszenz, tatsächlich wirksam sind. Die Autoren fanden teilweise positive Ergebnisse hinsichtlich kognitiver Leistungen für Reminiszenz und Realitätsorientierung. Die Evidenz für die Effektivität der berücksichtigten Verfahren in der Betreuung von Patienten mit Demenz war insgesamt jedoch unzureichend. Es fehlen bisher adäquate Interventionsstudien mit angemessenem methodischen Design, um die Wirksamkeit pflegerischer Betreuungskonzepte tatsächlich nachweisen zu können.

Eine andere, nicht minder wichtige Thematik sind Arzthaftpflichtfragen in der Behandlung von geriatrischen Patienten. Lucke et al. schildern aus der Arbeit der Schlichtungsstelle der norddeutschen Ärztekammern Fälle aus dem Grenzgebiet der Geriatrie und Unfallchirurgie. Sie zeigen exemplarisch 4 Fälle auf, im Bemühen, die Häufigkeit von Rechtsstreitigkeiten bei der Behandlung geriatrischer Patienten zukünftig zu verringern und auf wichtige Behandlungsfehler hinzuweisen.

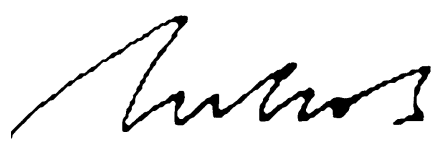

Prof. Dr. Th. Nikolaus

\section{Korrespondenzadresse}

Prof. Dr. Th. Nikolaus

Geriatrische Klinik gGmbH, Universtität Ulm

Zollernring 26, $89073 \mathrm{UIm}$

thorsten.nikolaus@bethesda-ulm.de
Carl-Albrecht Haensch,

Wolfgang Jost (Hrsg.)

\section{Das autonome Nervensystem}

Kohlhammer-Verlag 2010

394 S., 79.00 EUR

Die Beteiligung des autonomen Nervensystems an verschiedenen neurologischen Erkrankungen führt im klinischen Alltag häufig zu diagnostischen und therapeutischen Herausforderungen, jedoch existiert derzeit auf dem deutschsprachigen Büchermarkt kein umfassendes Nachschlagewerk. „Das autonome Nervensystem", herausgegeben von C.A. Haensch und W. Jost, zwei ausgewiesenen Experten auf dem Gebiet, schließt diese Lücke und bietet umfangreiche Informationen sowohl aus grundlagenorientierter wissenschaftlicher, als auch aus praxisnaher klinischer Sicht.

Das Buch gliedert sich in 19 Kapitel und beginnt mit einer detaillierten Aufstellung über Anatomie und Physiologie des autonomen Nervensystems. In zwei weiteren Kapiteln folgt eine praxisorientierte Erläuterung zu klinischer Untersuchung und speziellen Untersuchungsmethoden. Der Hauptteil des Buches widmet in logischem Aufbau allen neurologisch relevanten Krankheitsbildern ein eigenes Kapitel, in dem von erfahrenen Experten des jeweiligen Feldes eine ausführliche, jedoch stets präzise und praxisorientierte Abhandlung erfolgt. Abgerundet werden die einzelnen Kapitel durch ein kurzes Fazit für die Praxis mit den wichtigsten diagnostischen und therapeutischen Empfehlungen. Klare und übersichtliche Abbildungen illustrieren anschaulich besonders wichtige Aspekte. Trotz der Vielzahl unterschiedlicher Autoren gelingt im Text ein einheitliches Format, das durchwegs für eine sehr gute Verständlichkeit dieser ja zum Teil äußerst komplexen Thematik sorgt.
„Das autonome Nervensystem“ ist derzeit der einzige im deutschsprachigen Raum erhältliche klinische Leitfaden zur vegetativen Funktionsdiagnostik. Aufgrund dieser Einzigartigkeit, aber auch bei hoher Aktualität und Informationsfülle ist das Buch uneingeschränkt empfehlenswert für jedes vegetative Labor und alle wissenschaftlich und klinisch am Vegetativum Interessierten. Es bleibt zu hoffen, dass das Buch auf dem leicht in Vergessenheit geratenen Gebiet des vegetativen Nervensystems neue Impulse setzen und ein breiteres klinisches Interesse wecken kann.

Tobias Winkler (München) 